\title{
Rhabdomyosarcoma Histology Classification using Ensemble of Deep Learning Networks
}

\author{
Saloni Agarwal \\ University of Texas at Dallas \\ Richardson, Texas, United States \\ saloni.agarwal1@utdallas.edu
}

Ovidiu Daescu

University of Texas at Dallas

Richardson, Texas, United States

daescu@utdallas.edu

\author{
Mohamedelfatih Eltigani \\ Osman Abaker \\ University of Texas at Dallas \\ Richardson, Texas, United States \\ m.abaker@utdallas.edu
}

Donald A. Barkauskas

Keck School of Medicine of the

University of Southern California

Los Angeles, California, United States

dbarkauskas@childrensoncologygroup.org

\author{
Xinyi Zhang \\ University of Texas Southwestern \\ Medical Center \\ Dallas, Texas, United States \\ xinyi.zhang@utsouthwestern.edu \\ Erin R Rudzinski \\ Seattle Children's Hospital \\ Seattle, Washington, United States \\ erin.rudzinski@seattlechildrens.org
}

\author{
Patrick Leavey \\ University of Texas Southwestern \\ Medical Center \\ Dallas, Texas, United States \\ patrick.leavey@utsouthwestern.edu
}

\begin{abstract}
A significant number of machine learning methods have been developed to identify major tumor types in histology images, yet much less is known about automatic classification of tumor subtypes. Rhabdomyosarcoma (RMS), the most common type of soft tissue cancer in children, has several subtypes, the most common being Embryonal, Alveolar, and Spindle Cell. Classifying RMS to the right subtype is critical, since subtypes are known to respond to different treatment protocols. Manual classification requires high expertise and is time consuming due to subtle variance in appearance of histopathology images. In this paper, we introduce and compare machine learning based architectures for automatic classification of Rhabdomyosarcoma into the three major subtypes, from whole slide images (WSI). For training purpose, we only know the class assigned to a WSI, having no manual annotations on the image, while most related work on tumor classification requires manual region or nuclei annotations on WSIs. To predict the class of a new WSI we first divide it into tiles, predict the class of each tile, then use thresholding with soft voting to convert tile level predictions to WSI level prediction. We obtain $\mathbf{9 4 . 8 7 \%}$ WSI tumor subtype classification accuracy on a large and diverse test dataset. We achieve such accurate classification at $5 \mathrm{X}$ magnification level of WSIs, departing from related work, that uses $20 \mathrm{X}$ or $10 \mathrm{X}$ for best results. A direct advantage of our method is that both training and testing can be
\end{abstract}

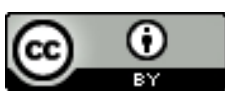

This work is licensed under a Creative Commons Attribution International 4.0 License. BCB '20, September 21-24, 2020, Virtual Event, USA

(C) 2020 Copyright held by the owner/author(s).

ACM ISBN 978-1-4503-7964-9/20/09.

https://doi.org/10.1145/3388440.3412486

performed much faster computationally due to the lower image resolution.

\section{CCS CONCEPTS}

- Computing methodologies $\rightarrow$ Computer vision; Ensemble methods; Neural networks; • Applied computing $\rightarrow$ Bioinformatics.

\section{KEYWORDS}

Histology, Deep Learning, Rhabdomyosarcoma, ensemble.

\section{ACM Reference Format:}

Saloni Agarwal, Mohamedelfatih Eltigani Osman Abaker, Xinyi Zhang, Ovidiu Daescu, Donald A. Barkauskas, Erin R Rudzinski, and Patrick Leavey. 2020. Rhabdomyosarcoma Histology Classification using Ensemble of Deep Learning Networks. In Proceedings of the 11th ACM International Conference on Bioinformatics, Computational Biology and Health Informatics (BCB '20), September 21-24, 2020, Virtual Event, USA. ACM, New York, NY, USA, 10 pages. https://doi.org/10.1145/3388440.3412486

\section{INTRODUCTION}

Rhabdomyosarcoma (RMS) is a pediatric soft tissue sarcoma that can occur in any anatomic site and counts as one of the ten most common childhood cancers [8] [26] [34] [29]. The most common locations of this tumor are head, neck, genitourinary tract and extremities. RMS has three major subtypes [30], Embryonal Rhabdomyosarcoma (ERMS), Alveolar Rhabdomyosarcoma (ARMS), and Spindle Cell/Sclerosing Rhabdomyosarcoma (SCRMS). The International World Health Organization (WHO) classification of Rhabdomyosarcoma has three risk groups-spindle cell as superior, embryonal as intermediate, and alveolar as unfavorable. A recent study [30] on a large dataset confirms WHO's classification system of RMS for risk stratification. The three types of RMS are shown in Figure 1. Different types of RMS are treated with different therapies. 


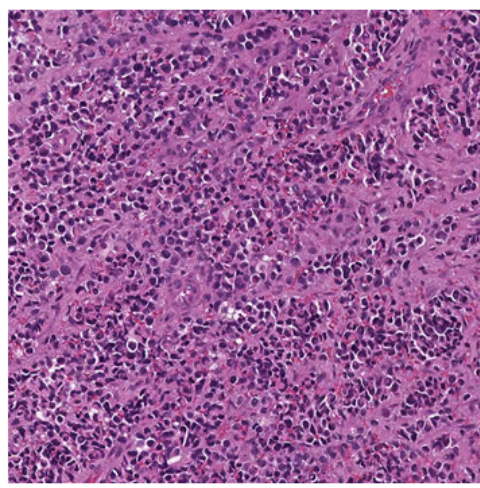

(a) Alveolar Rhabdomyosarcoma (ARMS)

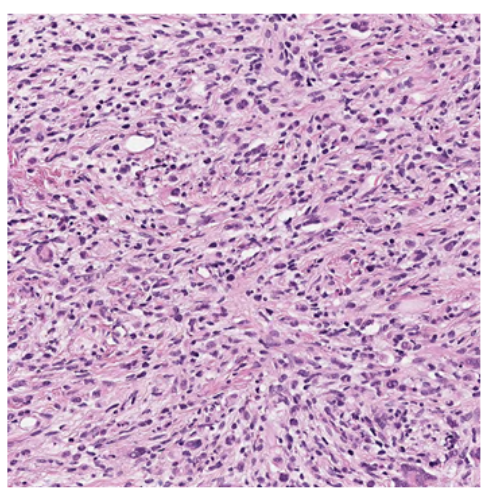

(b) Embryonal Rhabdomyosarcoma (ERMS)

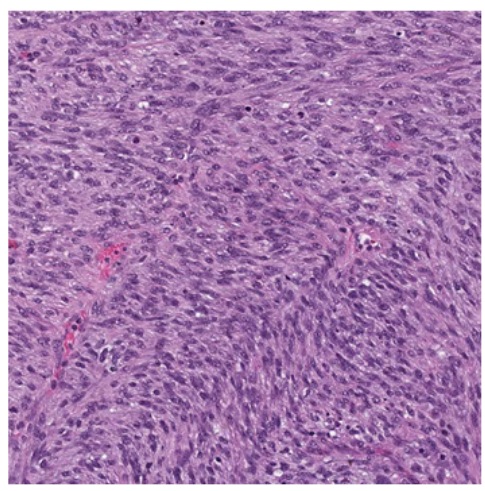

(c) Spindle Cell Rhabdomyosarcoma (SCRMS)

Figure 1: Different types of Rhabdyomasarcoma (RMS)

ARMS requires the most aggressive treatment, followed by ERMS, and then SCRMS. Thus, diagnosing the RMS in the correct subtype is critical for determining best treatment and improving outcome. However, RMS shows a plethora of variability in histology images, with intermixed features between subtypes, which makes recognizing the subtype a very challenging task, even for well trained pathologists [31] [26].

Currently, classification of RMS from histology involves inspection of histology slides by trained pathologists. The defining characteristic of RMS tumor is demonstration of skeletal muscle lineage, while the subtypes have slightly different histology features that are used for classification [31]. ARMS are of two types-classic pattern and solid pattern. Classic ARMS tumor cells have round and uniform nuclei. Solid ARMS contains cells with round nuclei arranged in sheets. ERMS, most common subtype of RMS, has itself four subtypes: typical, dense, botryoid and spindle cell pattern Typical pattern ERMS are dense cellular regions. Dense pattern ERMS is characterized by central or angulated nuclei. Botryoid pattern ERMS contains linear aggregates of tumor cells. Spindle cells have oval and blunted central nuclei. Dense pattern ERMS closely resembles ARMS [31] [26] and pathologists can often misclassify them. An automated tool for classification of the three major RMS subtypes will help in avoiding misclassification, reduce pathologist's workload, and standardize classification by mitigating inter-observer variability in diagnosis. Yet, while a lot of work has been done in developing machine learning based methods to identify and quantify major tumor types in histology images, much less is known about automatic classification of tumor subtypes, a significantly harder problem.

Recent years have seen an exponential growth in the application of deep learning in histopathology assessment. Particularly, convolutional neural networks $(\mathrm{CNN})$ have shown very good performance in bio-imaging segmentation and classification [1]. They are at the core of state-of-the-art models for nuclei segmentation [24] [19], breast cancer detection in histology images [27] [2] [40], liver cancer segmentation, colorectal cancer classification and segmentation [6] [17], lung nodule segmentation, and many other [21] [41] challenges in histopathology imaging. CNNs can extract representative features from images and these features can be used as image descriptors for training predictive models. Driven by the success of $\mathrm{CNNs}$ in medical image analysis we use CNNs to extract features for classification of RMS into subtypes.

In this paper, we propose a carefully designed and tested twolevel pipeline for classification of RMS-tile level and WSI level. There are a few key merits and advantages associated with our work. Unlike most work in cancer segmentation and classification, our approach does not require tumor region or nuclei annotations on WSIs; instead it automatically learns differences in RMS subtypes from larger size tiles with associated WSI class label mapped from the WSI from which the tile was extracted. We analyze WSIs at $5 \mathrm{x}$ magnification, which is uncommon in CNN based histology studies. Most existing studies use 20X or 10X magnification instead [11] [3]. We inferred through discussion with expert pathologists that larger size tiles $(1024 \times 1024$ pixels) at $5 x$ magnification would contain sufficient tumor nuclei to allow for pattern identification and correct class prediction. For tile-level classification, we use an ensemble of individually trained $\mathrm{CNN}$ models. Features are extracted from these CNN models and a multi-layer perceptron (MLP) is then trained on them. With the end goal of classifying WSIs, we then propose an approach to convert tile-level classification results into WSI-level classification results using thresholding of soft votes from tile prediction. A key contribution is our extensive experimentation for selecting the best ensemble, threshold values, and voting models to correctly classify the WSIs. Our experiments, on one of the largest and most diverse dataset available for RMS, show that some ensemble models have consistently good performance across threshold levels.

We conclude by recommending the MLP ensemble of CNN model, which shows remarkable performance. To the best of our knowledge, this is the first automated pipeline for histology imaged based subtype classification for RMS.

\section{RELATED WORK}

In most of the current state-of-the-art methods [11] [3] [27] [40], high magnification of the slide is used for analyzing histopathology 


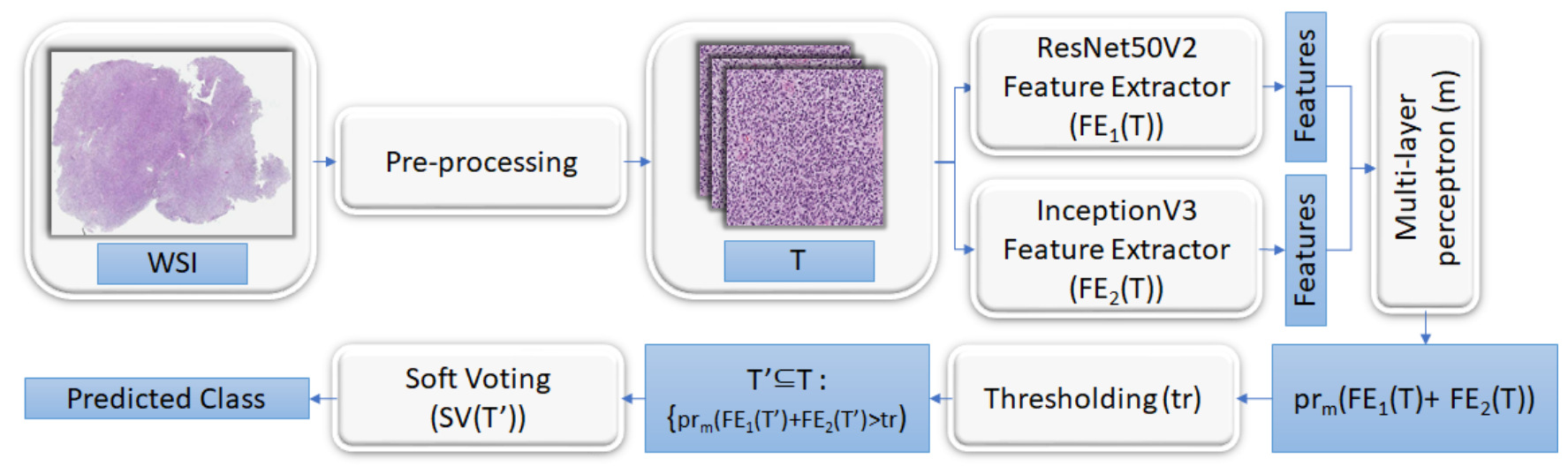

Figure 2: Ensemble of CNN model using thresholding for WSI classification

images. In some studies 10X (intermediate) magnification is used to increase the receptive field. In such research, the first step is to distinguish a region of interest (tumor region) from non-tumor regions using annotations on WSIs for model training. The properties of tissue are captured better at high resolution, therefore $10 \mathrm{X}$ and higher magnification is preferred for histology studies. Most of deep learning based histology studies use sliding window approach where models are trained for tile (size in hundreds $\times$ hundreds pixels at high magnification) level prediction. The ground truth for model training is obtained from hand annotated regions. The predictions from tile level are then aggregated for WSI level analysis [42] [3].

In histopathology image analysis, stain normalization is an essential step due to variation in stain intensities used during acquisition of WSI. Reinhard [28], Vahadane [39] and Macenko [22] are the most commonly used stain normalization techniques. In the Reinhard technique [28], target image color mean and standard deviation is linearly transformed to reference image. The major drawback of Reinhard technique is that multimodality of image is ignored and histology images are transformed using same normalization for background and foreground. Due to this stains are not separated properly. Vahadane [39] stain normalization is a technique that preserves all biological structures of target images. In this method, target image stain density maps are combined with stain color basis of a reference image. It results in only stain color changes while preserving tissue structure. This technique does not preserve all the color information of target images. In Macenko [22] stain normalization, stain vectors of the images are estimated using singular value decomposition of non-background pixels. It is based on the principle that color pixels in H\&E images are linear combinations of two stains. This method works well for quantitative analysis and we use this technique for stain normalization.

Traditionally, deep neural network architectures are inspired from human visual cortex and are analogous to neurons in human brain. They are made up of convolutional layers which transforms a $3 \mathrm{D}$ volume (input image-rgb channel $2 \mathrm{~d}$ array of pixels) using some parametric differential function to output a 3D volume. Using ideas of local receptive fields and shared weights, they are invariant to shifts and deformation. Once the features are detected using convolutional layers, they are subsampled in pooling layer. The pooling layer is followed by fully connected layers for final classification of input images into various classes. There has been rapid progress in CNN based image classification since 2012 [18], when a deep convolutional neural network was used to classify approximately 1.2 million images in 1000 classes. As compared to models developed after 2012, AlexNet [18] was relatively simple model with five convolutional layers and three fully connected layers, having approximately 62 million trainable parameters. After the success of AlexNet, there was an improvement in model architecture and a second family of networks emerged named VGG [33] network. In these models large filters from AlexNet were replaced with layers of smaller filters having same receptive field. There are various versions of this network like VGG16, VGG 19, etc., differing in number of layers used in architectural design.

ResNet [12] architecture was introduced to handle vanishing gradient problem in deep convolutional neural networks. These networks contain skip connections which help in propagating gradients through deeper networks. The most commonly used ResNet architectures are ResNet50 and ResNet101. Inception [37] model is another family of models made up of inception blocks containing multiple size filters arranged in parallel. Inception blocks help in capturing variable sizes of features from an image instead of simply being deeper with similar filter size. DenseNet [13] is another model architecture with every layer having access to all of its previous layer feature maps. One major difference between ResNet and DenseNet is that DenseNets do not sum the output feature maps with input feature maps but instead concatenate them before propagating features to next layer. InceptionResNet [36] is another very successful model architecture developed by Google. In this model architecture, inception blocks are connected with residual connections. We experimented with ResNet50V2 [12], DenseNet121 [13], InceptionV3 [37] and InceptionResNetV2 [36] models in this study due to fundamental differences in their model architecture. These architectures have been successfully used for histology image analysis in related work [41] [5] [7] [15] [10]. More recent computer vision models are known [38] [43], however they might not apply well in the pathology imaging domain. For example, even though 
InceptionReNetV2 is a more recent computer vision model, its performance on our RMS dataset is inferior to that of earlier models, such as ResNet50V2 and InceptionV3.

Due to the large number of parameters present in CNN models, a large dataset is needed for training a model. Transfer learning is a popular technique in which a pre-trained model can be adopted for a task of interest. A pre-trained model can be used as feature extractor. In this method, fully connected layers from already trained models are removed and intermediate features are used to train a machine learning classifier or a new set of fully connected layers. In this technique, only the new classifier, or the last fully connected layers are trained. Another way of using pre-trained models is by fine-tuning, where pre-trained model's original fully connected layers are replaced with new fully connected layers and the entire set of model weights are retrained on the new dataset. Transfer learning has shown better performance for medical image analysis than training a model from scratch [32]. It solves two major challenges in medical image analysis-limited medical data availability and medical domain knowledge understanding for identifying representative features. We use model fine-tuning for transfer learning in this study because it reduced model training time significantly while capturing relevant image features.

The ensemble approach [14] [44] [5] [7] [9] [15] [16] [23] refers to combining various base models together for improved prediction over individual models. Average voting is a common technique of ensemble deep learning models. In this technique, average of softmax class probabilities from different base deep learning models are used for class prediction. Majority voting is another method where the class is predicted by counting votes from base deep learning models and the class corresponding to maximum vote is the ensemble predicted class. Building a meta learner using base learners is another popular method of deep learning based ensembles. In this approach a meta learner, like random forest, is trained on features extracted from individually trained base models. Ensemble models have proved to provide superior results in various occasions. For example, in CAMELYON16 challenge for detection of lymph node metastases [5], the award winning approach was an ensemble of two GoogleNet models. In CAMELYON17 challenge, the top results were obtained by an ensemble of three ResNet101 models while the second entry used a single ResNet101 model. Results from [4] show that the use of ensemble of models can help in correcting individual predictions through voting. Because of these benefits of the ensemble approach, we decided to combine base model predictions using ensembles.

\section{METHODOLOGY}

\subsection{Dataset}

Through a Children's Oncology Group (COG) approved protocol (ARST18B4-Q) we examined 344 WSIs from diagnostic tissue biopsies of patients enrolled on Rhabdomyosarcoma specific COG prospective clinical trials. Each WSI image is associated with one of three RMS subtypes, ARMS, ERMS, and SCRMS. These WSIs were further reviewed by an expert pathologist in our team and 222 WSIs were assigned the same class label as the one provided by the diagnosing institutional pathologist for the individual patient. These 222 WSIs formed our dataset and had the following class

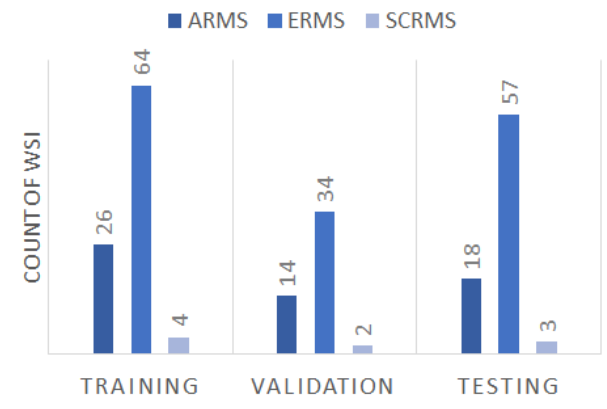

Figure 3: Plot of WSI data distribution across three classes

distribution: 58 ARMS, 155 ERMS, and 9 SCRMS. We divided the dataset into three separate sets for training, validation, and testing, and ensured that the test set was never seen by the classifier during training and fine-tuning. We sampled 94 training WSIs, 50 validation WSIs, and 78 testing WSIs from the 222 WSI dataset, while keeping the contribution of each class proportionally the same across subsets. The classwise WSI distribution is shown in Figure 3.

\subsection{Data Pre-processing}

The average size of a WSI in the dataset is $38,892 \times 29,964$ pixels. In order to process such large images we partition them into tiles. Initially, we experimented rigorously with tile size of $300 \times 300$ pixels at 10X magnification for RMS classification but none of the models converged. Tiles of relatively small size at $10 \mathrm{X}$ magnification seemed to lack information for capturing nuclei arrangement patterns. We then considered 5X magnification and created tiles of size $1024 \times 1024$ pixels to get a larger view of tissue. Moreover, pathologists informed us they usually start by looking at RMS WSIs at 5X magnification, to get a global view of the tissue. They can classify approximately $90 \%$ of RMS cases at this resolution and inspect the uncertain cases at higher magnification, like 10X.

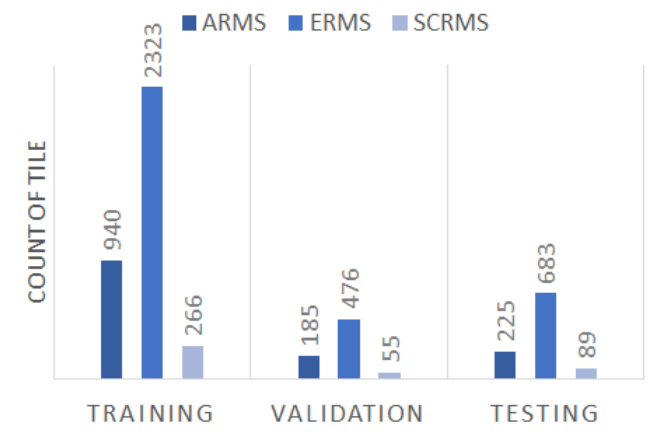

Figure 4: Plot of tile data distribution across three classes

Randomly selecting tiles from a WSI will result in many tiles without any relevant tissue region present in them. Therefore, we pre-process the WSIs for tile generation. We segment the tissue from WSI by generating 1/32 factor downscaled image. Hysteresis 
thresholding along with color-based filtering is used to get segmented tissue mask from downscaled image. We then split the WSIs into tiles of size $1024 \times 1024$ and assign a score based on tissue percentage and color properties. Tiles with score more than 85 on a scale of 1 to 100 are picked to form the dataset. We color normalized the generated tile images using Macenko stain normalization [22]. We choose a random image from training set as reference image for Macenko stain normalization of entire tile dataset. The tile dataset distribution for three subsets across various classes can be seen in Figure 4. As we can see there, after preprocessing the tile dataset is highly class imbalanced. We balance the training and validation subsets across the three classes by flipping and rotating images. Similar number of images in each class of the training subset implies equivalent contribution of classes in the loss function.

\subsection{Individual model training}

Four well known CNN architectures, ResNet50V2, DenseNet121, InceptionV3 and InceptionResNetV2 were chosen from the pool of available $\mathrm{CNN}$ models based on their performance on ImageNet classification, number of trainable parameters, and model architecture. In order to train such huge models, with millions of parameters, we use transfer learning. The models trained using transfer learning are already aware of image defining features and they learn specific tumor class features from the training dataset. We fine-tuned models pre-trained on ImageNet by removing the top fully connected layers and adding a dense layer of three neurons with softmax activation function. Weights of the model trained on ImageNet dataset are used as initial weight and entire network weights are updated at each gradient step. Each model is trained separately on balanced training data with same hyperparameters and categorical cross entropy objective function. We use adam optimizer with beta $1=0.9$, beta $2=0.999$, and $10 e^{-5}$ initial learning rate with decay after each epoch. We choose 8 or 16 batch size depending on the memory consumption of the model and available GPU memory. We trained the models for five epochs and saved the weight at epochs where performance increased on balanced validation dataset. The last saved weights are from the models performing best on validation dataset and we use them for testing purpose.

\subsection{Ensemble model}

The four individual models have their own shortcomings with respect to shape and texture in input images. In order to take advantages of individual base classifier, we built ensemble models to improve overall prediction. We built two sets of ensembles: ensemble of four CNNs and ensemble of two CNNs. In four CNNs ensemble, we use all four models-ResNet50V2, DenseNet121, InceptionV3, and InceptionResNetV2, while in ensemble of two CNNs we use the two individual best performing models. ResNet50V2 and InceptionV3 demonstrated best performance, as stated in Section 4, so we use them in ensemble of two. We combine $\mathrm{CNN}$ models to create ensembles using two main approaches-Probabilistic Approach and ML based Approach. Briefly, the probabilistic approach does not require any training and makes prediction by integrating outputs from individual models, while in the ML approach we extract features from last pooling layer of trained base CNNs and use them as input for ensemble classifier.
3.4.1 Probabilistic Approach: In this approach we ensemble the $\mathrm{CNN}$ models during test time. We combine the predictions of individual models in three ways: voting, soft voting, and most confident. Individual models predict the probability of an image belonging to three classes in the softmax layer and the class with highest probability is the predicted output. In voting approach, we count the number of models predicting each class and the class with maximum votes is the ensemble output. In soft voting approach instead of counting votes for each class, we average the predicted probability from the individual models for each class and the class having highest average probability is the ensemble output. Most confident approach predicts the class with maximum probability across different individual models. These approaches are mathematically represented in equations below.

$$
\begin{aligned}
& \mathrm{V}(t)=\operatorname{argmax}\left(\sum_{m \in \text { Models }} 1_{\operatorname{argmax}}\left(\operatorname{pr}_{m}(t)\right)\right) \\
& \mathrm{SV}(t)=\operatorname{argmax}\left(\sum_{m \in \text { Models }} \operatorname{pr}_{m}(t)\right) \\
& \operatorname{MC}(t)=\operatorname{argmax}\left(\max \left(\forall_{m \in \text { Models }} \operatorname{pr}_{m}(t)\right)\right)
\end{aligned}
$$

In these equations, $m$ represents individual CNN model from a set of models (Models). The predicted class probabilities for a tile $t$ from model $m$ is $\mathrm{pr}_{m}(t)$. In voting approach $(\mathrm{V}(t))$ we first compute the sum of one hot encoded predicted class from each model. The sum gives votes for each class by the models. Then, argmax over the sum results in the class having highest votes. In soft voting approach $(\mathrm{SV}(t))$, we sum predicted probability from each model $m$ instead of one hot encoded predicted class and get the predicted output by argmax over the sum. In most confident approach $(\mathrm{MC}(t))$, we predict the class where probability for the class is highest among all the models.

3.4.2 ML based Approach: We use three different ML models to create ensembles from features extracted by CNN models-Support vector Machine (SVM), Random Forest (RF), and Multi-layer perceptron (MLP). The scheme for ML based ensemble is shown in Figure 2. The Support Vector Machine (SVM) [35] classifiers are generally employed in problems with large number of features. They are not affected by outliers and work best if the classes are separable. We concatenate the features generated from base CNN models and feed them to a one-versus-rest multiclass SVM classifier for three class classification. The RMS classification task is relatively complex so we use a radial basis kernel. We used the scikit-learn machine learning library in python for building ensemble machine learning models. We used the default gamma value, which is $1 /$ (number of features $\times$ variance of features), and the default cost parameter of 1.0 for SVM models.

We build the second ensemble with a Random Forest classifier. RF models are resistant towards overfitting and can handle highly correlated features. A Random Forest (RF) model [20] averages prediction of decision trees built on randomly sampled features on random training data points. Two parameters are most important while building a random forest model: the number of features considered in each fork of the trees and the number of random trees assembled for model building. We keep these parameters to default 


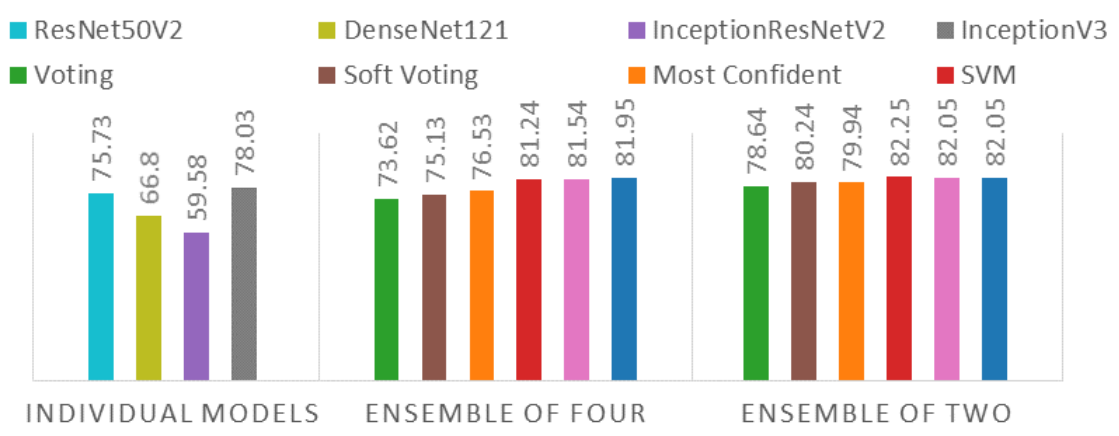

Figure 5: Plot of tile level classification accuracy for individual models, ensemble of four base models using different approaches, and ensemble of two base models using different approaches.

from scikit-learn's implementation of random forest, where 100 trees are formed using all the features.

For the third ensemble, we modify the head of individual CNNs and unite them using a two hidden layer multi-layer perceptron [25]. A two-layer multilayer perceptron is a universal estimator provided there is a sufficient number of neurons in hidden layers. A multilayer perceptron (MLP) can separate non-linear data and consists of several layers of neurons. In a typical MLP, all neurons in preceding layers are connected with neurons in following layers. We built MLP models with 64 and 32 neurons in first and second hidden layer, respectively, using Adam optimizer with 0.0001 learning rate.

\subsection{WSI Classification}

WSIs are divided into tiles and tiles are classified using the models described earlier. We convert tile predictions into WSI level prediction by using voting, soft voting, and most confident approach to aggregate predictions from tiles belonging to respective WSI.

Individual CNN models and all the variations of ensembles are inspected for WSI level analysis. We get class wise prediction probability for each tile from individual CNN models and ML based ensembles (SVM, RF and MLP) but we don't get class wise probabilities from probabilistic approach ensembles (voting, soft voting and most confident). Hence, we compute class probabilities from probabilistic approach based ensembles as follows:

$$
\begin{aligned}
\operatorname{VTP}(t) & =\frac{1}{M} \sum_{m \in \text { Models }} 1_{\operatorname{argmax}}\left(\operatorname{pr}_{m}(t)\right) \\
\operatorname{SVTP}(t) & =\frac{1}{M} \sum_{m \in \text { Models }} \operatorname{pr}_{m}(t) \\
\mathrm{P}(t) & =\max \left(\forall_{m \in \text { Models }} \operatorname{pr}_{m}(t)\right) \\
\mathrm{P}_{\mathrm{idx}}(t) & =\operatorname{argmax}\left(\forall_{m \in \text { Models }} \operatorname{pr}_{m}(t)\right) \\
\operatorname{MCTP}(t) & = \begin{cases}\mathrm{P}(t) & : \text { idx }=\mathrm{P}_{\mathrm{idx}}(t) \\
\frac{1-\mathrm{P}(t)}{2} & : \text { otherwise }\end{cases}
\end{aligned}
$$

We calculate tile probabilities using voting $(\operatorname{VTP}(t))$ by normalizing the calculated class votes for a tile $(t)$ over the total number of models $(M)$ in the ensemble. Similarly, we normalize the average predicted class probabilities from models $(m)$ over the total number of models $(M)$ in the ensemble for soft voting tile prediction $(\operatorname{SVTP}(t))$. In order to calculate tile probabilities for each class using most confident $(\operatorname{MCTP}(t))$ approach, we assign the highest predicted probability $(\mathrm{P}(t))$ to its class $\left(\mathrm{P}_{\mathrm{idx}}(t)\right)$ and distribute the remaining probabilities $(1-\mathrm{P}(t))$ equally among the other two classes.

However, few tiles in the dataset might not contain significant tumor region in them and they should not be included in WSI class prediction. Tiles with lower prediction probabilities have lower confidence. Inferior confidence of a tile could be due to insignificant tumor region present in them. Hence, we employ thresholding for WSI level analysis at $40 \%, 50 \%, 60 \%, 70 \%, 80 \%, 90 \%$ and $95 \%$ tile level prediction probability thresholds. The basic idea behind thresholding is to eliminate tiles with prediction probability lower than the threshold value in calculating WSI level classification accuracy. If we start with a set of $T$ tiles in the dataset (from $w$ WSIs), using thresholding we get a subset of tiles $T^{\prime}$ (from $n$ WSIs) satisfying the threshold value (tr). Note, $w-n$ WSIs are not being classified due to thresholding. We calculate WSI level prediction using voting, soft voting, and most confident approach on $T^{\prime}$ tiles. Let us say $l$ WSIs are correctly classified out of total $w$ WSIs. Then the WSI level classification accuracy of the dataset is the ratio of the WSIs correctly classified $(l)$ out of total number of WSIs $(w)$.

\subsection{Proposed Model Architecture}

The proposed model architecture is shown in Figure 2. A WSI is sent as input to the model and RMS subtype class is predicted as output. The WSI is first pre-processed, where tissue region is segmented, and tiles are extracted and normalized. We obtain a set of tiles $T$ for the given WSI. These tiles are provided to trained ResNet50V2 and InceptionV3 for feature extraction. We obtain features sets $\mathrm{FE}_{1}(T)$ and $\mathrm{FE}_{2}(T)$ from respective models after applying feature extraction (FE) on the tile set $T$. Further, we concatenate the features $\left(\mathrm{FE}_{1}(T)+\mathrm{FE}_{2}(T)\right)$ and send as input to the trained multi-layer perceptron model $(m)$. This model returns class-wise probabilities $\left(\operatorname{pr}_{m}\left(\mathrm{FE}_{1}(T)+\mathrm{FE}_{2}(T)\right)\right)$ for tiles in set $T$. We apply thresholding to the predicted class probabilities of tiles in set $T$, with threshold value tr equal to $80 \%$. We obtain a subset of tiles $T^{\prime}$ having predicted class probability greater than the threshold value tr. We apply soft voting (SV) on tile subset $T^{\prime}$ and the class corresponding to the highest probability is the predicted RMS subtype class output for the WSI. 

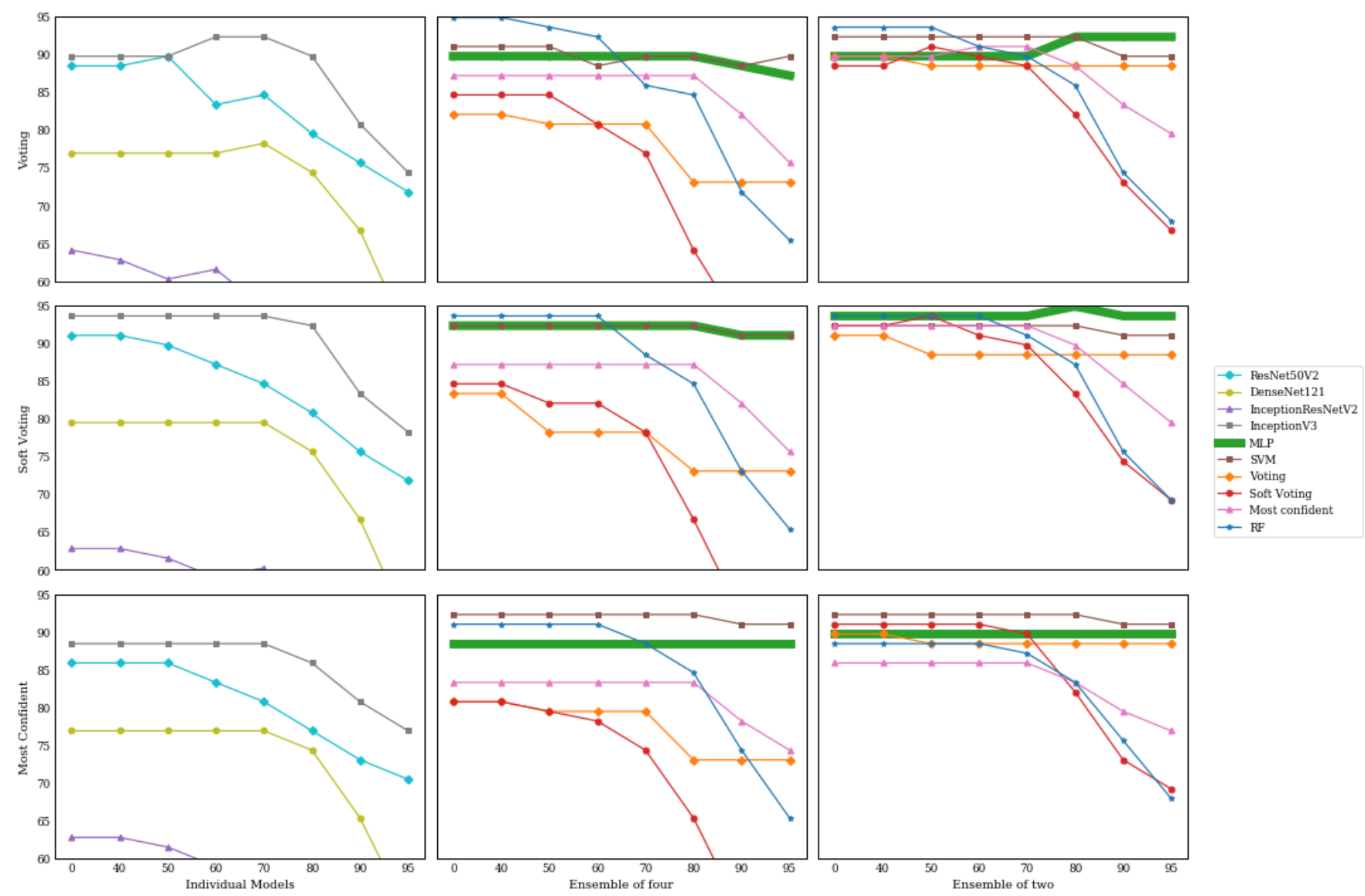

Figure 6: Plot of WSI level classification accuracies at various threshold values for individual models, ensemble of four CNNs, and ensemble of two CNNs using different approaches where voting, soft voting, and most confident are used for tile to WSI level classification.

\section{RESULTS}

We compare the performance of individual models, ensemble of two CNNs, and ensemble of four CNNs for tile level classification and WSI level classification on testing dataset. We use accuracy for checking model performance on tile classification, with the accuracy value given by the percentage of correctly classified tiles. For measuring model performance on WSI level classification we use testing dataset accuracy as well, given by percentage of correctly clasified WSIs. For WSI level prediction, we analyze the results at various threshold values for tile classification, using voting, soft voting, and most confident approach to convert tile level predictions to WSI level prediction. We conclude that the best performing model is MLP ensemble of two CNNs, where WSI level classification accuracy is calculated using soft voting at a threshold value of $\mathbf{8 0 \%}$, with $\mathbf{8 2 . 0 5 \%}$ tile classification accuracy and $\mathbf{9 4 . 8 7 \%}$ WSI level classification accuracy on the test dataset.

Tile level performance: Figure 5 shows performance of individual models, ensemble of four $\mathrm{CNN}$ models and ensemble of two CNN models. The first subplot exhibits performance of four individual CNN models-ResNet50V2, DenseNet121, InceptionResNetV2 and InceptionV3. We notice in the first subplot that InceptionV3 has highest tile classification accuracy of $78.03 \%$, followed by ResNet50V2 with $75.73 \%$ tile classification accuracy. The other two models-DenseNet121 (66.8\%) and InceptionResNetV2 (59.58\%), have significantly lower tile level prediction accuracies. Next two subplots show performance of ensemble of four CNNs and ensmble of two CNNs using probabilistic ensembles (voting, soft voting and most confident) and ML based ensembles (SVM, RF and MLP). Overall, we observe that ensembles perform better than individual models. Further, ensemble of two CNN models have superior performance as compared to ensemble of four CNN models. Individually, ResNet50V2 and InceptionV3 has better classification accuracies as compared to DenseNet121 and InceptionResNetV2 and combining them results in better performance in ensemble of two models as compared to ensemble of four models.

We can also observe that ML based ensembles (SVM, RF and MLP) have superior performance as compared to probabilistic ensembles (voting, soft voting and most confident) in both ensemble of four and ensemble of two subplots. Training machine learning 
models on features extracted from CNN models favors tile classification. Further, these differences between probability based and ML based ensemble predictions are larger in ensemble of four plots as compared to ensemble of two. Hence, presence of under performing models in ensemble of four has more negative impact on probabilitybased ensembles as compared to ML based ensembles. The best tile prediction accuracy is $82.25 \%$ using ensemble of two CNNs with SVM classifier. Overall, the ML based ensembles of two CNN models have stable and comparable performance (SVM-82.25\%, $\mathrm{RF}-82.05 \%$ and MLP-82.05\%).

WSI level performance: WSI level test dataset classification accuracies for individual models, ensemble of four models, and ensemble of two models are plotted in Figure 6, using voting, soft voting, and most confident approaches for converting tile level classification to WSI level classification. Left, middle, and right columns in the plot show performance of individual models, ensemble of four CNNs, and ensemble of two CNNs, respectively. Among individual models, we have performance of ResNet50V2, DesnseNet121, InceptionResNetV2, and InceptionV3 shown in first column. The second and third columns contain predictions using probabilistic ensembles (voting, soft voting, and most confident) and ML based ensembles (SVM, RF, and MLP) from two and four CNNs respectively. The $x$-axis in all the subplots represents the cutoff threshold values. The best individual model performance is $93.59 \%$ by InceptionV3 using soft voting approach at initial threshold values $(0 \%$, $40 \%, 50 \%, 60 \%$ and $70 \%$ ). Aligning with tile level prediction, we observe that among individual models InceptionV3 performs better followed by ResNet50V2, DesnNet121, and InceptionResNetV2, over all three strategies and all threshold values.

The accuracy of individual models is outperformed by ensemble models, with the overall best WSI classification performance of $94.87 \%$ using the MLP ensemble of two CNNs with soft voting, at $80 \%$ threshold value. We note that the same accuracy is obtained using voting by RF ensemble of four at $0 \%$ and $40 \%$ threshold values. Like tile prediction, we observe ensembles perform better than individual models for WSI prediction as well. Further, we also observe ensemble of two having similar top performance as ensemble of four but at different threshold values. Soft voting predictions are slightly better than voting followed by most confident, for most of the models at all thresholds.

In ensemble of two and four we can observe that probabilistic based models have inferior performance to ML based ensembles at all thresholds. RF performs well at low threshold values, but are highly unstable and the performance decreases with increase in threshold values. This is because RF computes probabilities for each class based on voting from constituent trees, which is similar to probabilistic approach. In all three strategies for ensemble of two and four, SVM and MLP perform reasonably well and both of them are quite stable. Since soft voting and ensemble of two perform better overall then other approaches, and we observe MLP having an edge over SVM in the corresponding subplot, we propose MLP as the model of choice.

In most cases, WSI prediction accuracy decreases at $90 \%$ and $95 \%$ threshold values. This is because as we increase the threshold, the number of tiles below the threshold increases and they are eliminated, in turn reducing the number of WSIs used for classification (some of the WSIs have no representative tiles in the resulting set).
This results in subsequent decrease of correctly classified WSIs $(l)$ and overall accuracy. It is interesting to notice that InceptionV3 with predictions by voting has better performance when threshold values are in the middle (60\% and $70 \%)$. We can observe a similar behaviour for MLP ensemble of two with soft voting at threshold value of $80 \%$. Thresholding with $70 \%-80 \%$ cutoff value helps in improving accuracy of well performing models.

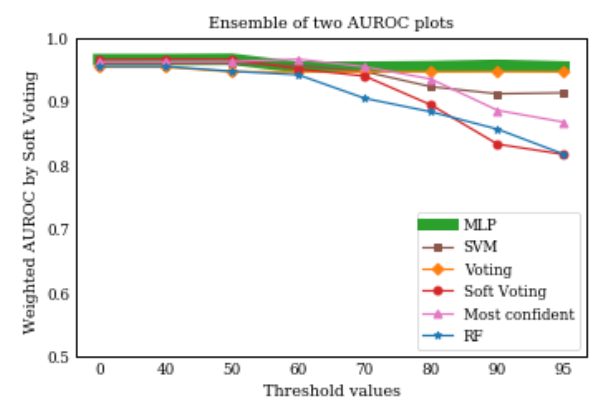

Figure 7: Weighted AUROC values for various ensembles of two using soft voting at different threshold values.

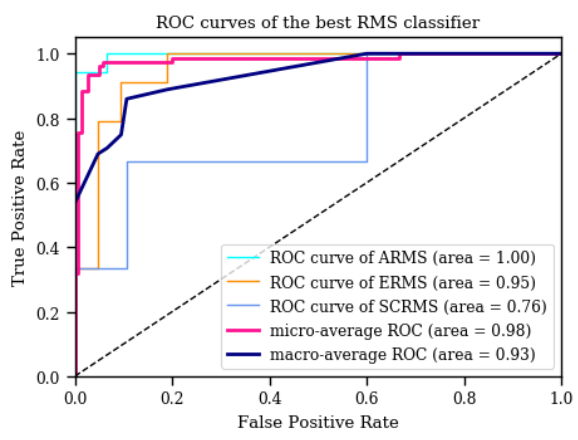

Figure 8: ROC plot for each class and their macro and micro averages using MLP ensemble of two using soft voting approach at $80 \%$ threshold value.

We also analyze the models' WSI level classwise performance using the weighted area under the receiver operating characteristic curve (AUROC) value at various thresholds for the individual models and ensembles. It follows similar trend as the WSI level classification accuracy. Figure 7 shows the plot of weighted AUROC at different threshold values of the ensembles of two models using soft voting. In this plot, the MLP based ensemble has a very stable AUROC value. Figure 8 depicts classwise AUROC values at $80 \%$ threshold value using MLP soft voting ensemble of two model. This model has 0.98 micro-average AUROC value and 0.93 macro-average AUROC value. The AUROC value for ARMS and ERMS classes is very high, but the SCRMS class is comparatively low. We find that tiles from SCRMS WSIs are misclassified as ERMS. According to the pathologist review, SCRMS are of three types, one being spindle predominant ERMS. It isn't easy to differentiate this type of SCRMS from ERMS class based on histology images, and molecular data is essential for correct classification of such WSIs. 


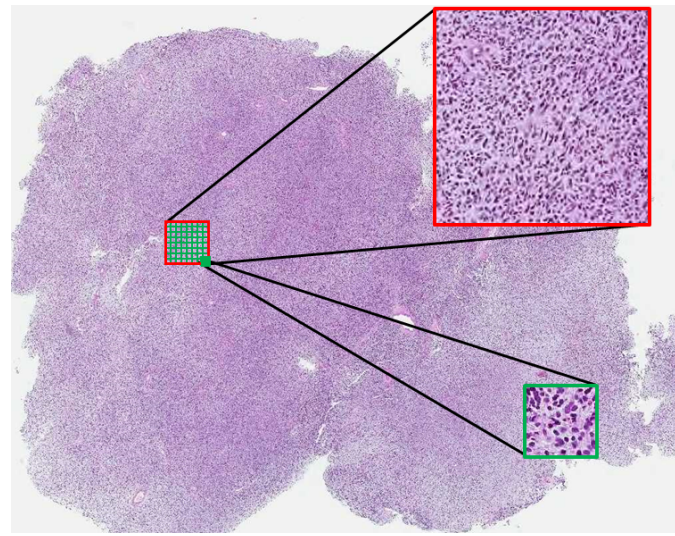

Figure 9: Comparing receptive field of a tile generated at $5 \mathrm{X}$ magnification (in red border) with tile generated at $10 \mathrm{X}$ magnification (in green border).

\section{DISCUSSION}

In this paper, we employ different techniques for tile and WSI level classification of RMS data. It is interesting to note the effect of thresholding, ensembles, learning for WSI classification, conversion strategy, processing at lower magnification and the lifting effect of tile level classification on WSI level classification.

Effect of thresholding: In Figure 6 we see that well performing models-InceptionV3 in individual models, SVM and MLP in ensembles-are stable until above $80 \%$ threshold values, and their performance decreases after that. In fact, in certain cases, like InceptionV3 with voting and MLP in ensemble of two CNN, using voting and soft voting, we observe that performance increases at $70 \%-80 \%$ threshold values. We infer thresholding at $70 \%-80 \%$ improves distinguishing capability of powerful models. On the other hand, increase in threshold value decreases the WSI classification accuracy of weak models. It can be associated with increase in number of WSIs not classified and subsequent decrease in correctly classified WSIs. Weak performing models might have lower class prediction probabilities for many tiles which are eliminated at higher thresholds.

Effect of building ensemble models: As shown in Figure 5 and Figure 6, ensemble models perform better than individual models on both tile classification and WSI classification. Ensembles helps in overcoming deficiencies of individual model. From the differences in ensemble of two and four CNN models, we observe that combination of well performing individual models helps in creating better ensembles, as in ensemble of two CNN. Since an ensemble is a mixture of the individual models used to create it, inclusion of under performing models has a negative impact on an ensemble performance, as in ensemble of four CNN.

Effect of learning for WSI classification: We observe in both ensemble of two and four CNNs that learned ML models like SVM, $\mathrm{RF}$, and MLP perform better than probabilistic voting, soft voting, and most confident for WSI classification. This shows that extracting features from individual models and learning class differences results in better classifiers than simply combining individual models using their probabilistic outcomes. It also suggests that features extracted from individual models have class defining properties and individual models exhibit differences in RMS subtype features captured. Due to variability in features captured by individual models, creating ensembles leads to combining more class defining features and better performance.

Effect of conversion strategy: While building ensembles, we directly get class wise probabilities using soft voting from models. In voting and most confident ensemble strategy, we compute class wise probabilities by adopting votes and highest probability. The results from most confident ensembles are quite stable and decreases after $90 \%$ threshold value. On the other hand, soft voting ensembles are highly affected by thresholding and very unstable.

Effect of processing at lower magnification: The WSI prediction results using tiles at $5 \mathrm{X}$ with $1024 \times 1024$ pixel size shows great success. Due to high receptive field of these tiles as shown in Figure 9 , lesser number of tiles are generated from a WSI. Comparatively, it captures 64 times more tissue region then captured by a tile at $10 \mathrm{X}$ magnification of $256 \times 256$ pixel size. Lesser number of tiles has a direct impact on training and testing time and makes processing and classification of new tiles much faster.

Effect of tile level prediction on WSI level prediction: As expected, tile level prediction has direct impact on WSI level predictions. Models with higher tile level accuracy lead to better WSI level classification, especially without thresholding. Further, at higher threshold, more tiles are removed for WSI classification and the number of WSIs classified is reduced.

\section{CONCLUSION}

We propose a novel architecture for RMS subtype histopathology classification using multi-layer perceptron ensemble of features extracted from ResNet50V2 and InceptionV3 CNN models with thresholding and soft voting. The proposed model has an accuracy of $94.87 \%$ for WSI image classification into RMS subtypes. We proposed thresholding for WSI prediction and observed thresholding helps in improving prediction of well performing models. It supports our hypothesis that removal of tiles with low confidence favors WSI level classification. We also observe that histopathology classification of RMS subtypes is possible with high accuracy at $5 \mathrm{X}$ magnification. This study is a first step towards a large project on long term survival prediction of RMS patients. Motivated by this study's success in histopathology classification of RMS subtypes, in future work we will integrate molecular data with histopathology for RMS subtype analysis and long term outcome prediction.

\section{ACKNOWLEDGEMENTS}

This work was supported by Children's Cancer Fund. The material from COG was supported through NCTN Operations Center Grant U10CA180886, NCTN SDC Grant U10CA180899 and COG Biospecimen Bank Grant U24CA196173.

\section{REFERENCES}

[1] S. Agarwal, R. R. Hallac, R. Mishra, C. Li, O. Daescu, and A. Kane. 2018. Image Based Detection of Craniofacial Abnormalities using Feature Extraction by Classical Convolutional Neural Network. In 2018 IEEE 8th International Conference on Computational Advances in Bio and Medical Sciences (ICCABS). 1-6.

[2] Guilherme Aresta, Teresa Araújo, Scotty Kwok, Sai Saketh Chennamsetty, Mohammed Safwan, Varghese Alex, Bahram Marami, Marcel Prastawa, Monica 
Chan, Michael Donovan, et al. 2019. Bach: Grand challenge on breast cancer histology images. Medical image analysis 56 (2019), 122-139.

[3] Harish Babu Arunachalam, Rashika Mishra, Ovidiu Daescu, Kevin Cederberg, Dinesh Rakheja, Anita Sengupta, David Leonard, Rami Hallac, and Patrick Leavey. 2019. Viable and necrotic tumor assessment from whole slide images of osteosarcoma using machine-learning and deep-learning models. PloS one 14, 4 (2019).

[4] Peter Bandi, Oscar Geessink, Quirine Manson, Marcory Van Dijk, Maschenka Balkenhol, Meyke Hermsen, Babak Ehteshami Bejnordi, Byungjae Lee, Kyunghyun Paeng, Aoxiao Zhong, et al. 2018. From detection of individual metastases to classification of lymph node status at the patient level: the camelyon17 challenge. IEEE transactions on medical imaging 38, 2 (2018), 550-560.

[5] Babak Ehteshami Bejnordi, Mitko Veta, Paul Johannes Van Diest, Bram Van Gin neken, Nico Karssemeijer, Geert Litjens, Jeroen AWM Van Der Laak, Meyke Hermsen, Quirine F Manson, Maschenka Balkenhol, et al. 2017. Diagnostic assessment of deep learning algorithms for detection of lymph node metastases in women with breast cancer. Fama 318, 22 (2017), 2199-2210.

[6] Dmitrii Bychkov, Nina Linder, Riku Turkki, Stig Nordling, Panu E Kovanen, Clare Verrill, Margarita Walliander, Mikael Lundin, Caj Haglund, and Johan Lundin. 2018. Deep learning based tissue analysis predicts outcome in colorectal cancer. Scientific reports 8, 1 (2018), 1-11.

[7] Sai Saketh Chennamsetty, Mohammed Safwan, and Varghese Alex. 2018. Classification of breast cancer histology image using ensemble of pre-trained neural networks. In International conference image analysis and recognition. Springer, 804-811.

[8] Ramzi Dagher and Lee Helman. 1999. Rhabdomyosarcoma: an overview. The oncologist 4, 1 (1999), 34-44.

[9] Mehmet Günhan Ertosun and Daniel L Rubin. 2015. Automated grading of gliomas using deep learning in digital pathology images: A modular approach with ensemble of convolutional neural networks. In AMIA Annual Symposium Proceedings, Vol. 2015. American Medical Informatics Association, 1899.

[10] Carlos A Ferreira, Tânia Melo, Patrick Sousa, Maria Inês Meyer, Elham Shakibapour, Pedro Costa, and Aurélio Campilho. 2018. Classification of breast cancer histology images through transfer learning using a pre-trained inception resnet v2. In International Conference Image Analysis and Recognition. Springer, 763-770.

[11] Arkadiusz Gertych, Zaneta Swiderska-Chadaj, Zhaoxuan Ma, Nathan Ing, Tomasz Markiewicz, Szczepan Cierniak, Hootan Salemi, Samuel Guzman, Ann E Walts, and Beatrice S Knudsen. 2019. Convolutional neural networks can accurately distinguish four histologic growth patterns of lung adenocarcinoma in digital slides. Scientific reports 9, 1 (2019), 1-12.

[12] Kaiming He, Xiangyu Zhang, Shaoqing Ren, and Jian Sun. 2016. Identity mappings in deep residual networks. In European conference on computer vision. Springer 630-645.

[13] Gao Huang, Zhuang Liu, Laurens Van Der Maaten, and Kilian Q Weinberger 2017. Densely connected convolutional networks. In Proceedings of the IEEE conference on computer vision and pattern recognition. 4700-4708.

[14] Cheng Ju, Aurélien Bibaut, and Mark van der Laan. 2018. The relative performance of ensemble methods with deep convolutional neural networks for image classification. Journal of Applied Statistics 45, 15 (2018), 2800-2818.

[15] Sara Hosseinzadeh Kassani, Peyman Hosseinzadeh Kassani, Michal J Wesolowski, Kevin A Schneider, and Ralph Deters. 2019. Classification of histopathological biopsy images using ensemble of deep learning networks. arXiv preprint arXiv:1909.11870 (2019)

[16] Daisuke Komura and Shumpei Ishikawa. 2018. Machine learning methods for histopathological image analysis. Computational and structural biotechnology journal 16 (2018), 34-42.

[17] Bruno Korbar, Andrea M Olofson, Allen P Miraflor, Catherine M Nicka, Matthew A Suriawinata, Lorenzo Torresani, Arief A Suriawinata, and Saeed Hassanpour. 2017. Deep learning for classification of colorectal polyps on whole-slide images. fournal of pathology informatics 8 (2017).

[18] Alex Krizhevsky, Ilya Sutskever, and Geoffrey E Hinton. 2012. Imagenet classification with deep convolutional neural networks. In Advances in neural information processing systems. 1097-1105.

[19] Jiahui Li, Shuang Yang, Xiaodi Huang, Qian Da, Xiaoqun Yang, Zhiqiang Hu, Q Duan, Chaofu Wang, and Hongsheng Li. 2019. Signet ring cell detection with a semi-supervised learning framework. In International Conference on Information Processing in Medical Imaging. Springer, 842-854.

[20] Andy Liaw, Matthew Wiener, et al. 2002. Classification and regression by randomForest. $R$ news 2, 3 (2002), 18-22.

[21] Jiahao Lu, Nataša Sladoje, Christina Runow Stark, Eva Darai Ramqvist, Jan Michaél Hirsch, and Joakim Lindblad. 2019. A Deep Learning based Pipeline for Efficient Oral Cancer Screening on Whole Slide Images. arXiv preprint arXiv:1910.10549 (2019)

[22] Marc Macenko, Marc Niethammer, James S Marron, David Borland, John T Woosley, Xiaojun Guan, Charles Schmitt, and Nancy E Thomas. 2009. A method for normalizing histology slides for quantitative analysis. In 2009 IEEE International Symposium on Biomedical Imaging: From Nano to Macro. IEEE, 1107-1110.
[23] Pim Moeskops, Max A Viergever, Adriënne M Mendrik, Linda S De Vries, Manon JNL Benders, and Ivana Išgum. 2016. Automatic segmentation of MR brain images with a convolutional neural network. IEEE transactions on medical imaging 35, 5 (2016), 1252-1261.

[24] P. Naylor, M. Laé, F. Reyal, and T. Walter. 2017. Nuclei segmentation in histopathology images using deep neural networks. In 2017 IEEE 14th International Symposium on Biomedical Imaging (ISBI 2017). 933-936.

[25] Sankar K Pal and Sushmita Mitra. 1992. Multilayer perceptron, fuzzy sets, classifiaction. (1992).

[26] David M Parham and Frederic G Barr. 2013. Classification of rhabdomyosarcoma and its molecular basis. Advances in anatomic pathology 20, 6 (2013), 387.

[27] Alexander Rakhlin, Alexey Shvets, Vladimir Iglovikov, and Alexandr A Kalinin. 2018. Deep convolutional neural networks for breast cancer histology image analysis. In International Conference Image Analysis and Recognition. Springer, 737-744.

[28] Erik Reinhard, Michael Adhikhmin, Bruce Gooch, and Peter Shirley. 2001. Color transfer between images. IEEE Computer graphics and applications 21, 5 (2001), 34-41.

[29] Erin R Rudzinski, James R Anderson, Yueh-Yun Chi, Julie M Gastier-Foster, Caroline Astbury, Frederic G Barr, Stephen X Skapek, Douglas S Hawkins, Brenda J Weigel, Alberto Pappo, et al. 2017. Histology, fusion status, and outcome in metastatic rhabdomyosarcoma: A report from the Children's Oncology Group. Pediatric blood \& cancer 64, 12 (2017), e26645.

[30] Erin R Rudzinski, James R Anderson, Douglas S Hawkins, Stephen X Skapek, David M Parham, and Lisa A Teot. 2015. The World Health Organization classification of skeletal muscle tumors in pediatric rhabdomyosarcoma: a report from the Children's Oncology Group. Archives of pathology \& laboratory medicine 139, 10 (2015), 1281-1287.

[31] Erin R Rudzinski, Lisa A Teot, James R Anderson, Julie Moore, Julia A Bridge, Frederic G Barr, Julie M Gastier-Foster, Stephen X Skapek, Douglas S Hawkins, and David M Parham. 2013. Dense pattern of embryonal rhabdomyosarcoma, a lesion easily confused with alveolar rhabdomyosarcoma: a report from the Soft Tissue Sarcoma Committee of the Children's Oncology Group. American journal of clinical pathology 140, 1 (2013), 82-90.

[32] Chuen-Kai Shie, Chung-Hisang Chuang, Chun-Nan Chou, Meng-Hsi Wu, and Edward Y Chang. 2015. Transfer representation learning for medical image analysis. In 2015 37th annual international conference of the IEEE Engineering in Medicine and Biology Society (EMBC). IEEE, 711-714.

[33] Karen Simonyan and Andrew Zisserman. 2014. Very deep convolutional networks for large-scale image recognition. arXiv preprint arXiv:1409.1556 (2014).

[34] Stephen X Skapek, Andrea Ferrari, Abha A Gupta, Philip J Lupo, Erin Butler, Janet Shipley, Frederic G Barr, and Douglas S Hawkins. 2019. Rhabdomyosarcoma. Nature Reviews Disease Primers 5, 1 (2019), 1-19.

[35] Johan AK Suykens and Joos Vandewalle. 1999. Least squares support vector machine classifiers. Neural processing letters 9, 3 (1999), 293-300.

[36] Christian Szegedy, Sergey Ioffe, Vincent Vanhoucke, and Alexander A Alemi. 2017. Inception-v4, inception-resnet and the impact of residual connections on learning. In Thirty-first AAAI conference on artificial intelligence.

[37] Christian Szegedy, Vincent Vanhoucke, Sergey Ioffe, Jon Shlens, and Zbigniew Wojna. 2016. Rethinking the inception architecture for computer vision. In Proceedings of the IEEE conference on computer vision and pattern recognition. 2818-2826.

[38] Hugo Touvron, Andrea Vedaldi, Matthijs Douze, and Hervé Jégou. 2019. Fixing the train-test resolution discrepancy. In Advances in Neural Information Processing Systems. 8252-8262.

[39] Abhishek Vahadane, Tingying Peng, Shadi Albarqouni, Maximilian Baust, Katja Steiger, Anna Melissa Schlitter, Amit Sethi, Irene Esposito, and Nassir Navab. 2015. Structure-preserved color normalization for histological images. In 2015 IEEE 12th International Symposium on Biomedical Imaging (ISBI). IEEE, 1012-1015.

[40] Juan Vizcarra, Ryan Place, Li Tong, David Gutman, and May Dongmei Wang. 2019. Fusion In Breast Cancer Histology Classification. In Proceedings of the 10th ACM International Conference on Bioinformatics, Computational Biology and Health Informatics. 485-493.

[41] Jason W Wei, Laura J Tafe, Yevgeniy A Linnik, Louis J Vaickus, Naofumi Tomita, and Saeed Hassanpour. 2019. Pathologist-level classification of histologic patterns on resected lung adenocarcinoma slides with deep neural networks. Scientific reports 9,1 (2019), 1-8.

[42] Jason W Wei, Laura J Tafe, Yevgeniy A Linnik, Louis J Vaickus, Naofumi Tomita, and Saeed Hassanpour. 2019. Pathologist-level classification of histologic patterns on resected lung adenocarcinoma slides with deep neural networks. Scientific reports 9,1 (2019), 1-8.

[43] Qizhe Xie, Minh-Thang Luong, Eduard Hovy, and Quoc V Le. 2020. Selftraining with noisy student improves imagenet classification. In Proceedings of the IEEE/CVF Conference on Computer Vision and Pattern Recognition. 1068710698 .

[44] Ali Yazdizadeh, Zachary Patterson, and Bilal Farooq. 2019. Ensemble Convolutional Neural Networks for Mode Inference in Smartphone Travel Survey. IEEE Transactions on Intelligent Transportation Systems (2019). 